\title{
Preface for the Ursula Sonnewald Honorary Issue of Neurochemical Research
}

\author{
Arne Schousboe $^{1}$
}

Published online: 27 May 2017

(C) Springer Science+Business Media New York 2017

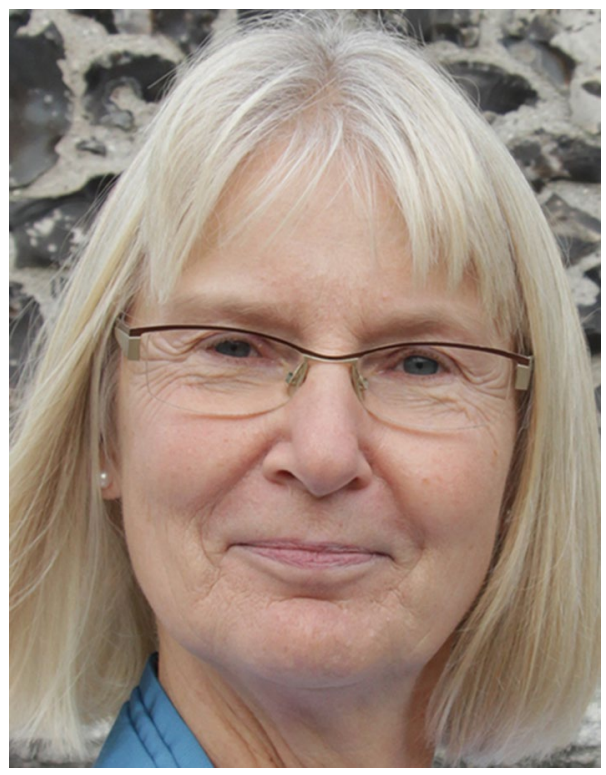

This special issue of Neurochemical Research contains manuscripts from a large number of neuroscientists throughout the world to honor Ursula Sonnewald for her outstanding contributions in refining the NMR technology, enabling detailed studies at the cellular level using ${ }^{13} \mathrm{C}$-labeled substrates to be performed; studies that have provided important new knowledge leading to a better understanding of brain energy metabolism.

Ursula Sonnewald, who was born in Germany, received her basic training in organic chemistry and obtained her

Arne Schousboe

arne.schousboe@sund.ku.dk

1 University of Copenhagen, Copenhagen, Denmark
Ph.D. in chemistry at the University of Saskatchewan, Saskatoon, Canada. She subsequently undertook post-doc positions at University of Utah, Salt Lake City, UT, USA, The Carlsberg Research Center, Copenhagen, Denmark, and The Brookhaven National Laboratory, Upton, New York, USA. After the completion of the post-doc periods, Ursula was employed as a Medicinal Chemist at the CNS Department, NOVO-Nordisk, Copenhagen, Denmark working on the development of anti-epileptic drugs. Other drug companies had previously worked along this line of research; investigating the possibility of using lipophilic analogs of the GABA-transport inhibitor nipecotic acid as anticonvulsant compounds in animal models of epilepsy. While successful in developing such GABA-transport inhibitors as effective anticonvulsants in the animal models, 
subsequent studies in humans had failed. In spite of this, Ursula, together with a group of behavioral pharmacologists at NOVO-Nordisk, synthesized $(R)-N$-[4,4-bis(3methyl-2-thienyl)but-3-en-1-yl]nipecotic acid which was eventually developed as the antiepileptic drug tiagabine; that for a number of years has been in clinical use to treat certain forms of epilepsy.

In 1990, Ursula decided for family reasons to move to a position at the Norwegian University of Science and Technology, Trondheim, Norway to work on brain metabolism using Nuclear Magnetic Resonance (NMR) spectroscopy as a tool. Prior to leaving Denmark, Ursula had consulted with me at the University of Copenhagen to discuss the possibility of using the NMR technology to study glucose metabolism in cultured neurons and astrocytes using $\left[{ }^{13} \mathrm{C}\right]$ glucose. I had expressed some skepticism due to the limited availability of cellular material from cell cultures and the lack of sensitivity of the NMR method, but Ursula insisted that we should try to carry out preliminary experiments. Fortunately doing so, turned out to be successful and based on the first set of results, we succeeded in demonstrating for the first time that astrocytes in culture had the ability to synthesize and release large amounts of citrate [1, 2]. This represented the start of a highly productive collaboration, which has lasted from 1991 until today, i.e. for well over 25 years. It is clear from several of the publications, included in this honorary issue of Neurochemical Research, that Ursula has managed to form collaborative networks with a large number of scientists in Europe, Asia, Australia and USA. These collaborations have resulted in numerous highly cited publications describing neuron-glial interactions in energy metabolism. Additionally, she has employed these techniques to study brain metabolism in a number of animal models of epilepsy, stroke, and schizophrenia, as well as in models of neurodegenerative diseases including Alzheimer's disease.
Ursula has also played a significant role in educating the next generation of neuroscientists working in the field of brain metabolism. Thus, both nationally in Trondheim and internationally at the University of Copenhagen, where a large number of $\mathrm{Ph} . \mathrm{D}$. students have received basic training in the use of NMR technology to study metabolic processes, both in vivo and in vitro using either intact brains or cultured brain cells. Some of these students have contributed papers to this special issue of Neurochemical Research honoring Ursula. She has also contributed to the development of research in brain metabolism; being actively involved in the organization of international meetings in the series "International Conferences of Brain Energy Metabolism" (ICBEM), both as a member of several of its Scientific Organizing Committees, as the main Conference Organizer of the Meeting in Trondheim 2001, and as the Key Note speaker at the most recent ICBEM meeting held last year in Hong Kong. Altogether, it is obvious that Ursula has had an instrumental impact on the advancement of studies of brain energy metabolism.

Arne Schousboe

Guest Editor

\section{References}

1. Sonnewald U, Westergaard N, Krane J, Unsgård G, Petersen SB, Schousboe A (1991) First direct demonstration of preferential release of citrate form astrocytes using ${ }^{13} \mathrm{C}$ NMR spectroscopy of cultured neurons and astrocytes. Neurosci Lett 128:235-239

2. Westergaard N, Waagepetersen HS, Belhage B, Schousboe A (2017) Citrate, a ubiquitous key metabolite with regulatory function in the CNS. Neurochem Res. doi:10.1007/ s11064-016-2159-7 